phương pháp điện châm, xoa bóp kết hợp vật lý trị liệu, Trường Đại học Y Hà Nội, Hà Nội.

2. H'ô Hữu Lương (2008). Đau thắt lựng và thoát vị đĩa đệm, Nhà xuất bản Y học, Hà Nội, tr 76 - 217.

3. C. Massullo(2017). I Guna Collagen medical device nella ripresa funzionale dopo traumi sportivi case reports.

4. Milani L. A new and refined injectable treatment for musculoskeletal disorder. Bioscaffold properties of collagen and its clinical use. Physiological Regulating Medicine 2010/1; 3-15. 2

5. Raychev I(2013). Efficacy and safety of combined treatment with GuNa MD - Lumbra and GuNa MD - Ishial Collagen injection in patients with lumbar disc hernation.

6. Tsai KS, Kao SY, Wang CY, Wang YJ, WangJP,
Hung SC. Type I collagen promotes proliferation and osteogenesis of human mesenchymal stem cells via activation of ERK and Akt pathways. J Biomed Mater Res A. 2010 Sep l;94(3):673-82.

7. Jeasen GJ., Ponte DJ., Kent BE. (1984). A preliminary report on the use of the Mc Kentzie protocol versus William protocol in the treatment of low back pain. Journal of Orthopaedic and Sport PhysicalcTherapy, Vol.6, No.2, pp 130 - 139.

8. J. Guitart Vela, J. Folch Ibáñez(2016-2017). Collagen MDs for chronic pain. Eficacy and tolerability in chronic treatment in 124 patients.

9. K. Pavelka, R. Svobodová, H. Jarošová (2012) MD-Lumbar, MD-Muscle and MD-Neural in the treatment of low back pain.

\title{
PHÂN TÍCH CHI PHÍ HIỆU QUẢ BẰNG MÔ HÌNH CỦA BUDESONIDE/FORMOTEROL KHI CẦN Ở NGƯỜI BỆNH HEN NHE TẠI VIÊTT NAM
}

\author{
Phạm Huy Tuấn Kiệt ${ }^{1}$, Lê Đặng Tú Nguyên², \\ Heleen van Haalen ${ }^{3}$, Trương Văn Đạt ${ }^{2}$, Lê Hồng Phương ${ }^{4}$, \\ Nguyễn Thị Hải Yến ${ }^{2}$, Nguyễn Thị Quỳnh $\mathrm{Nga}^{2}$
}

\section{TÓM TẮT}

Mục tiêu: Phân tích chi phí-hiệu quả của Budesonide/Formoterol khi cân so với Corticosteroid dang hít (ICS) liều thấp hàng ngày phối hợp với thuốc đồng vân beta giao cảm tác dung ngắn (SÄBA) khi cần ở người bệnh hen phế quản (HPQ) nhẹ tại Việt Nam. Phương pháp nghiên cứu: Mồ hình hóa sử dụng mô hình Markov gồm ba trạng thái chính: không có đợt cấp $\mathrm{HPQ}$, đợt cấp $\mathrm{HPQ}$ nặng, và tử vong. Nghiên cứu được thực hieên dưới quan điểm của cơ quan chi trả, khung thời gian chay mô hình là toàn thời gian sống với chu kỳ của mố hình là một tuân. Hiệu quả lâm sàng được trích xuất từ nghiên cứu SYGMA 2 và các thông số về chi phí được dựa trên một nghiên cứu gánh nặng bệnh tật tại Việt Nam và cơ sở dữ liệu của Cục Quản lý Dược. Kết quả mô hình được biểu thị dưới dạng chỉ số chi phí-hiệu quả tăng thểm (ICER), kết quả phân tích độ nhạy một chiều và phân tích độ nhay xác suất. Kết quả: Budesonide/Formoterol khi cần vượt trội so với phác đồ so sánh, giúp tránh được 0,44 đợt cấp HPQ, tiết kiệm 2.632.464 VNĐ chi phí điêuu trị và tăng $0,0006 \mathrm{QALY} s$ về mặt hiệu quả. Kết quả phân tích độ nhạy một chiều và độ nhạy xác suất đều khẳng định trong đa số trường hợp phân tích, Budesonide/Formoterol khi cân đều đạt chi phí-hiệu

\footnotetext{
${ }^{1}$ Trường Đại học Y Hà Nội

ªại học Y Dược Thành phố Hồ Chí Minh

${ }^{3}$ Công ty AstraZeneca Thụy Điên

${ }^{4}$ Công ty TNHH AstraZeneca Việt Nam

Chịu trách nhiệm chính: Nguyễn Thị Quỳnh Nga

Email: nguyenthiquynhnga@ump.edu.vn

Ngày nhận bài: 17.5.2021

Ngày phản biện khoa học: 29.6.2021

Ngày duyệt bài: 16.7.2021
}

quả. Kết luânn: Từ quan điểm của cơ quan chi trả, Budesonide/Formoterol khi cần tiết kiệm chi phí và tăng hiệu quả so với phác đồ ICS liều thấp phối hợp SABA khi cần về tính chi phí-hiệu quả tại Việt Nam.

Từ khóa: Budesonide/Formoterol, Hen phế quản nhẹ, Phân tích chi phí-hiệu quả

\section{SUMMARY \\ MODELLED COST-EFFECTIVENESS ANALYSIS OF AS-NEEDED BUDESONIDE/FORMOTEROL IN PATIENTS WITH MILD ASTHMA IN VIETNAM}

Objectives: Cost-effectiveness analysis of Budesonide/Formoterol as needed compared with daily low dose inhaled corticosteroids (ICS) in combination with short-acting beta agonists as needed (SABA) in patients with mild asthma in Vietnam. Research methods: A probabilistic Markov cohort model was developed including three main states: non-exacerbation, severe exacerbation, and death. This study was carried out from the payer perspective, with a lifetime horizon and one-week cycle. Clinical parameters were derived from SYGMA 2 trial and costs were extracted from a cost-of-illness study in Vietnam and Drug Administration of Vietnam database. Results were expressed as an incremental cost-effectiveness ratio (ICER), results of one-way sensitivity analysis and probabilistic sensitivity analysis. Results: On average, as needed Budesonide/Formoterol was associated with VND 2,632,464 cost savings, qualityadjusted life year (QALY) gains of 0.0006 and 0.44 exacerbations avoided versus ICS combined with asneeded SABA. Budesonide/Formoterol thus dominated the comparator regarding cost-effectiveness. The results of one-way sensitivity analysis and probabilistic sensitivity analysis confirmed the robustness of the 
basecase results. Conclusion: From the payer perspective, Budesonide/Formoterol as needed saved cost while increasing effectiveness relative to the lowdose ICS combined with as-needed SABA regime in Vietnam.

Keywords: Budesonide/formoterol, Costeffectiveness analysis, Mild asthma.

\section{I. ĐĂT VẤN ĐỀ}

Hen phế quản (HPQ) là một vấn đề y tế toàn câu nghiêm trọng có xu hướng gia tăng trong những thập niên vừa qua, đặc biệt là ở các nước có thu nhập thấp và trung bình. Trên toàn thế giới, HPQ là nguyên nhân tử vong đứng hàng thứ hai trong số các bệnh mãn tính đường hô hấp [1]. Tai Việt Nam, một nghiên cứu khảo sát tại 7 tỉnh thành đại diên cho 7 vùng sinh thái trên cả nước cho thấy tỷ lệ mắc HPQ ở người trưởng thành Việt Nam là $4,1 \%$ nhưng chỉ có $29,1 \%$ trong số này được điêu trị bằng liệu pháp dự phòng hen [2].

HPQ có thể gây ảnh hưởng xấu đến sức khỏe, công việc, kinh tế gia đình và xã hội. Do đó, cân có các biện pháp điều trị kiểm soát $\mathrm{HPQ}$ phù hợp nhằm giảm thiểu tình trạng tàn tật, tử vong liên quan đến $\mathrm{HPQ}$ và giảm bớt gánh nặng kinh tế cho người bệnh. Ở đối tượng người bệnh $\mathrm{HPQ}$ nhẹ, mặc dù các triệu chứng ít, việc kém tuân thủ liệu pháp duy trì thường xuyên như Corticoid liêuu thấp dạng hít (Inhaled Corticosteroids, ICS) làm tăng nguy cơ tăng bậc hen và tân suất xuất hiện cơn hen kịch phát cần được quan tâm [3]. Song song với đó, người bệnh sử dụng nhóm thuốc đồng vận beta giao cảm tác dụng ngắn (Short-Acting Beta Agonists, SABA) khi cân để cắt cơn, tuy nhiên SABA không thể phòng ngừa được tình trạng viêm đường thở và hạn chế xuất hiện cơn hen kịch phát trong tương lai [3].

Theo khuyến cáo của Bộ $Y$ tế trong chẩn đoán và điêu trị hen phế quản năm 2020, Budesonide/Formoterol là thuốc ưu tiên trong kiểm soát và cắt hơn hen ở người bệnh $\mathrm{HPQ}$ nhẹ. Các nghiên cứu thử nghiệm lâm sàng đã cho thấy đối với bệnh nhân HPQ nhẹ, Budesonide/Formoterol khi cân có hiệu quả lâm sàng không thua kém và mức độ an toàn tương đương ICS liêuu thấp (ngày 2 lần) phối hợp với SABA khi cân (ICS+SABA) $[3,4]$. Do đó, nghiên cứu phân tích kinh tế dược Budesonide/ Formoterol khi cần so với phác đồ phổ biến cho điều trị $\mathrm{HPQ}$ nhẹ sẽ góp phần cung cấp bằng chứng khoa học cho việc tối ưu hóa phác đồ điêu trị về cả hiệu quả và chi phí, từ đó đem lại chất lượng sống tốt nhất cho đối tượng người bệnh này. Nghiên cứu này được thực hiện nhằm mục tiêu phân tích chi phí-hiệu quả của
Budesonide/Formoterol khi cân so với liêu pháp ICS+SABA ở người bệnh HPQ nhẹ tại Việt Nam với ba mục tiêu cụ thể: (i) phân tích chi phí-hiệu quả; (ii) phân tích độ nhạy một chiều; (iii) phấn tích độ nhạy xác suất.

\section{II. ĐỐI TƯợNG VÀ PHƯƠNG PHÁP NGHIÊN CỨU}

2.1. Đối tượng nghiên cứu. Đối tượng nghiên cứu là quần thể người bệnh HPQ giả lập theo tiêu chuẩn đâu vào của nghiên cứu thử nghiệm lâm sàng ngẫu nhiên có đối chứng (RCT) SYGMA 2: bệnh nhân $\geq 12$ tuổi với chẩn đoán lâm sàng được ghi nhận là $\mathrm{HPQ}$ nhẹ $[3,4]$. Tuổi trung bình của dân số có quốc tịch Việt Nam trong SYGMA 2 là 38,2 ( $\pm 14,7)$, với tỷ lệ nữ là 55,9\%.

2.2. Phương pháp nghiên cứu. Nghiên cứu phân tích kinh tế dược bằng phương pháp mô hình hóa sử dụng mô hình Markov phù hợp với đặc điểm và diễn tiến của bệnh HPQ. Mô hình được xây dựng dựa trên dữ liệu trích xuất từ nghiến cứu RCT đa trung tâm đa quốc gia SYGMA 2, kết hợp với tham vấn ý kiến chuyên gia. Mô hình gồm ba trạng thái chính: không có đợt cấp $\mathrm{HPQ}$, đợt cấp $\mathrm{HPQ}$ nặng và tử vong. Đợt cấp HPQ năng được phân loại thành ba nhóm dựa trên việc quản lý bệnh nhân trong đợt cấp nặng: sử dụng glucocorticosteroids toàn thân (GCS) $\geq 3$ ngày, nhập viện cấp cứu (Emergency room, ER), và ER + GCS (Hình 1).

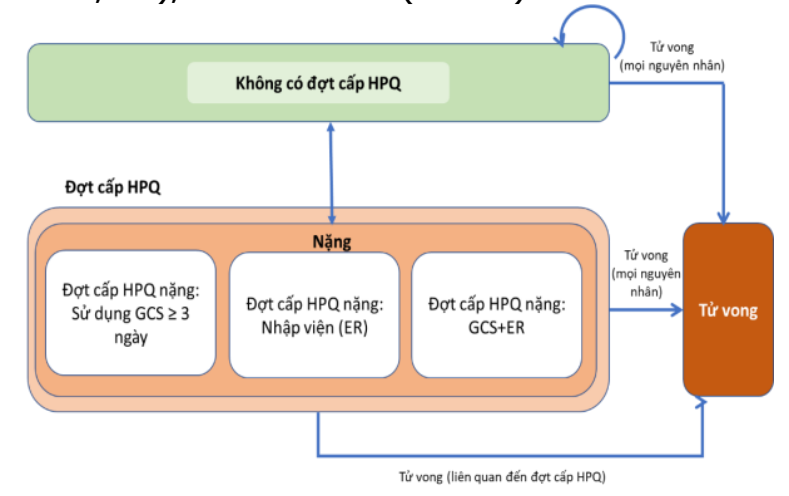

Hình 1. Cấu trúc mô hình Markov

Ghi chú: ER: nhâp viên câp cứu, GCS:

Glucocorticosteroids, GINA: Global Strategy for Asthma Management and Prevention.

Dữ liệu đâu vào của mô hình

Hiếu quả điều trí: Nghiên cứu SYGMA 2 là thử nghiệm mù đôi, ngẫu nhiên, đa trung tâm cho thây hiệu quả của Budesonide/Formoterol thông qua tỷ lệ cơn kịch phát hàng năm, thời gian đến cơn kịch phát đâuu tiên và chất lượng cuộc sống [3]. Nghiên cứu SYGMA 2 được thực hiện tại 25 quốc gia khác nhau trong đó có Việt Nam [4]. Tại Việt Nam, nghiên cứu được thực 
hiện tại tám bệnh viện và trung tâm y tế lớn trên cả ba miên Bắc, Trung và Nam [3]. Do đó, các thông số đầu vào mô hình bao gồm tỷ lệ đợt cấp nặng hàng năm, phân bổ chiến lược kiểm soát HPQ giữa các đợt cấp, tỷ lệ tăng bậc điều trị, và tỷ lệ xảy ra biến cố có hại của thuốc được trích xuất từ dữ liệu nghiên cứu này.

Chất lượng sống của người bệnh: Hệ số chất lượng sống được thu thập trong nghiên cứu SYGMA 2 với công cụ EQ-5D-5L [3,4]. Chỉ số hiệu quả về chất lượng sống của người bệnh sẽ được biểu thị bởi giá trị số năm sống tăng thêm có điều chỉnh bởi chất lượng sống (QALY Quality-adjusted Life Year).

Chi phí thuốc: Dữ liệu về chi phí thuốc được trích xuất từ cơ sở dữ liệu về giá thuốc của Cục Quản lý Dược Việt Nam [5]. Chi phí hàng ngày được tính toán từ tổng chi phí thuốc và liều lượng được chỉ định sử dụng hàng ngày của thuốc. Dữ liệu về số lần sử dụng mối ngày của từng nhóm điều trị được lấy từ nghiên cứu SYGMA $2[3,4]$.

Chi phí trực tiếp y tễ: Bao gồm chi phí xét nghiệm, chi phí chẩn đoán hình ảnh, chi phí thuốc, chi phí thủ thuật - phẫu thuật, chi phí vật tư, chi phí dịch vụ kỹ thuật, chi phí khám, chi phí vận chuyển và chi phí khác liên quan đênn điều trị duy trì $\mathrm{HPQ}$ ở các trạng thái không có đợt cấp $\mathrm{HPQ}$, đợt cấp $\mathrm{HPQ}$ nặng (với ba phân nhóm sử dụng glucocorticosteroids (GCS) toàn thân $\geq 3$ ngày, nhập viện cấp cứu (ER), ER + GCS). Dữ liệu về chi phí trực tiếp y tế được trích xuất từ nghiên cứu gần đây về phân tích chi phí bệnh tật của HPQ tại Việt Nam [6]. Nghiên cứu được thực hiện dưới quan điểm của cơ quan chi trả, tỷ lệ chiết khấu $3 \%$, thời gian chạy mô hình là toàn thời gian sống của người bệnh (70 năm), với chu kỳ của mô hình là một tuần. Chi phí có đơn vị là VND và giá trị năm 2020.

Phân tích chi phí-hiêu quả. Kết quả phân tích chi phí-hiệu quả là chi phí tăng thêm và hiệu quả tăng thêm của phối hợp Budesonide/ Formoterol so với phác đồ so sánh (ICS+SABA). Từ đó, chỉ số chi phí-hiệu quả tăng thêm ICER (Incremental cost-effectiveness ratio) được tính toán. Chỉ số ICER - chỉ số cho biết chi phí gia tăng trên một đơn vị hiệu quả gia tăng được ước tính theo công thức:

$$
\begin{aligned}
& \text { Chi phi } i_{\text {Budesonide/Formoterol }}-\text { Chi phíl ICS/SABA } \\
& \text { Hiệu quả }{ }_{\text {Budesonide/Formoterol }}-H i e \underline{u} u \text { quả }{ }_{\text {ICS/SABA }}
\end{aligned}
$$

Trong đó: ICS+SABA: Corticosteroids dạng hít liều thấp (ngày 2 lần) phối hợp với SABA khi cần.

Phân tích độ nhạy. Nghiên cứu sử dụng phương pháp phân tích độ nhạy một chiều và phân tích độ nhạy xác suất. Phân tích độ nhạy một chiều đánh giá tác động của từng biến số đển chỉ số chi phí-hiệu quả ICER. Dựa trên phân tích này, biểu đồ Tornado được thiết lập để mô tả mức độ tác động của các biến số lên giá trị ICER. Các biến số chính dự kiến ảnh hưởng đến chi phí-hiệu quả của mô hình so với ICS+SABA là: tỷ lệ đợt cấp HPQ nặng hàng năm, chi phí đợt cấp $H P Q$, chi phí trạng thái không có đợt cẩp HPQ hàng năm, liều dùng ICS, hệ số chất lượng sông của người bệnh có đợt cấp HPQ, tỷ lệ chiết khấu và tuổi đầu vào của mô hình.

Phân tích độ nhạy xác suất (PSA) đánh giá tác động đồng thời của nhiều biến số đến chi phí-hiệu quả của mô hình. Trong phương pháp phân tích độ nhạy xác suất PSA, mổi biến số đầu vào của mô hình đều có một mức độ bất định nhất định và được mô tả thông qua một phân phối xác suất. Nghiên cứu sử dụng 3000 vòng lặp Monte Carlo để ghi nhận các cặp giá trị chi phí và hiệu quả khác nhau. Kết quả sẽ được trình bày thông qua giá trị ước lượng điểm ICÉR kèm theo khoảng tin cậy $95 \%$, đường cong chấp nhận chi phí-hiệu quả và mặt phẳng chi phí-hiệu quả với đám mẩy ước lượng chỉ số ICER.

\section{KẾT QUẢ NGHIÊN CỨU}

3.1. Phân tích chi phí-hiệu quả. Dựa theo quan điểm của cơ quan chi trả, phân tích chi phíhiệu quả của Budesonide/Formoterol khi cần so với phác đồ ICS+SABA được trình bày ở bảng 3.1

Tổng số QALYs và chi phí của Budesonide/ Formoterol và phác đồ so sánh lần lượt là 21,0311 QALYs và 21,0305 QALYs; 23.399 .634 VNĐ và 26.032.098 VNĐ. So với phác đồ so sánh, Budesonide/Formoterol khi cần mang lại hiệu quả tăng thêm là 0,0006 QALYs [KTC 95\%:0,0011 đến 0,0023 ] đồng thời làm giảm chi phí điều trị 2.632.464 VND [KTC 95\%: -12.511.485 đến 7.716.163]. So sánh tính chi phí-hiệu quả, Budesonide/Formoterol khi cần vượt trội so với phác đồ ICS+SABA. Phân tích nền cũng cho thấy trong suốt vòng đời của người bệnh, số đợt cấp HPQ nặng khi sử dụng Budesonide/ Formoterol khi cần là 4,80 so với 5,24 đợt của phác đồ so sánh. Như vậy Budesonide/ Formoterol khi cần giúp tránh được 0,44 đợt cấp HPQ nặng. 
Bảng 3.1. Kêt quả phân tích chi phí-hiêuu quả

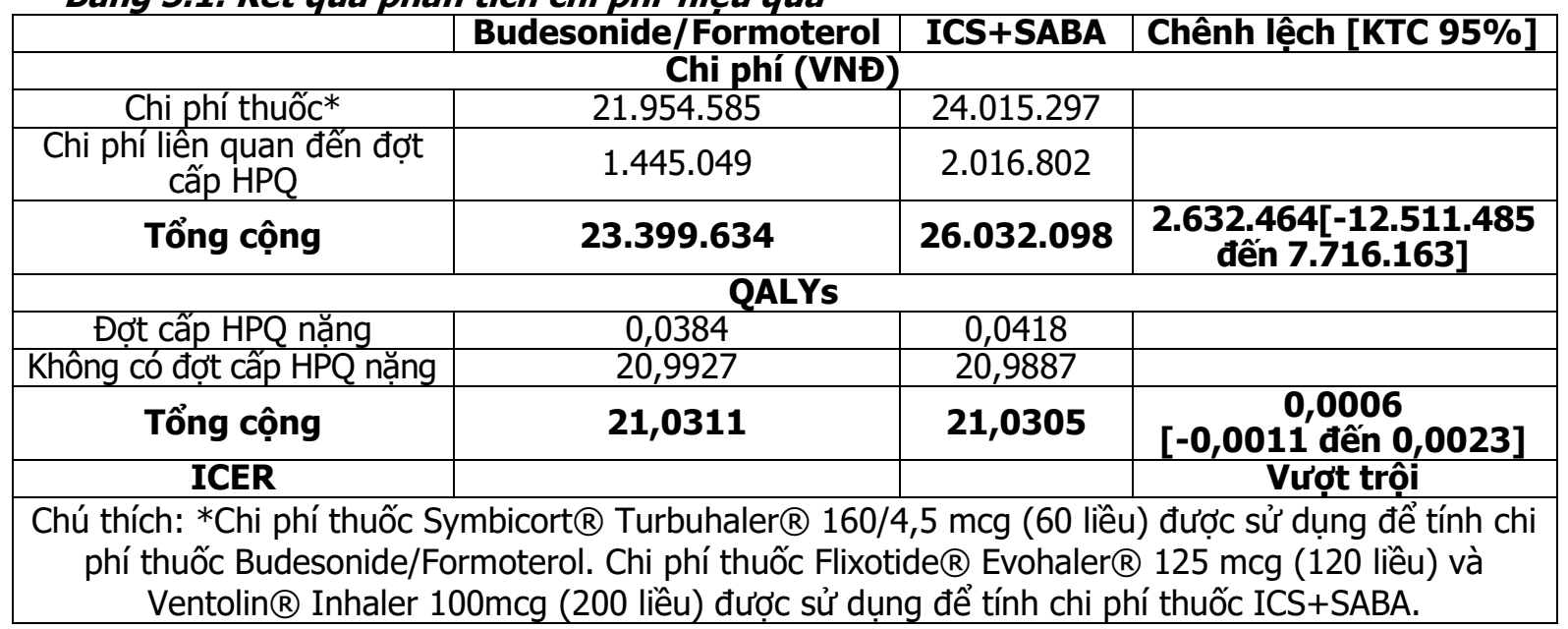

3.2. Phân tích độ nhạy một chiêu. Phân tích độ nhạy một chiều cho thãy các biến số có ảnh hưởng nhiều nhất đến giá trị ICER là tỷ lệ đợt cấp HPQ nặng hàng năm, tỷ lệ chiết khấu (cho chi phí và hiệu quả), và hệ số chất lượng sống của đợt cấp HPQ nặng. Trong hầu hết các trường hợp của phân tích độ nhạy một chiều, giá trị ICER của Budesonide/Formoterol vượt trội hơn so với phác đồ so sánh, ngoại trừ trường hợp thay đổi tỷ lệ đợt cấp HPQ nặng hàng năm (tăng số đợt cấp HPQ nặng đối với Budesonide/Formoterol hoặc giảm số đợt cấp HPQ nặng đối với phác đồ so sánh) (Hình 2).

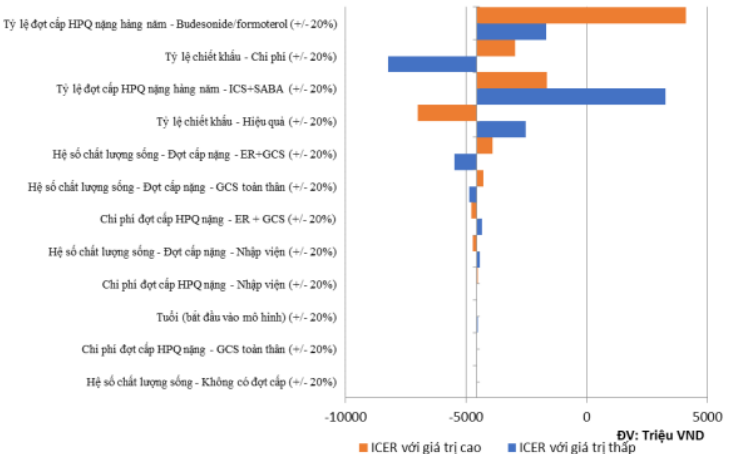

Hình 2. Biểu đồ Tornado - Phân tích độ nhay môt chiều

Chú thích: Giá trị của từng biến số được biến thiên trong khoảng $\pm 20 \%$ so với phân tích chi phí-hiệu quả ở bước trên. ER: nhập viện cấp cứu, HPQ: hen phế quản, ICS+SABA: Corticosteroids dạng hít liều thấp (ngày 2 lần) phối hợp với SABA khi cần, GCS: Glucocorticosteroid.

\subsection{Phân tích độ nhạy xác suất.}

Kết quả phân tích độ nhay xác suất PSA sử dụng phương pháp Monte-Carlo với 3.000 vòng lặp và được trình bày trong Hình 3 và Hình 4 . Kết quả trên mặt phẳng chi phí-hiệu quả của Budesonide/Formoterol khi cần so với ICS+SABA cho thấy trong đa số trường hợp, Budesonide/ Formoterol khi cần đều vượt trội so với phác đồ so sánh về tính chi phí-hiệu quả (Hình 3).

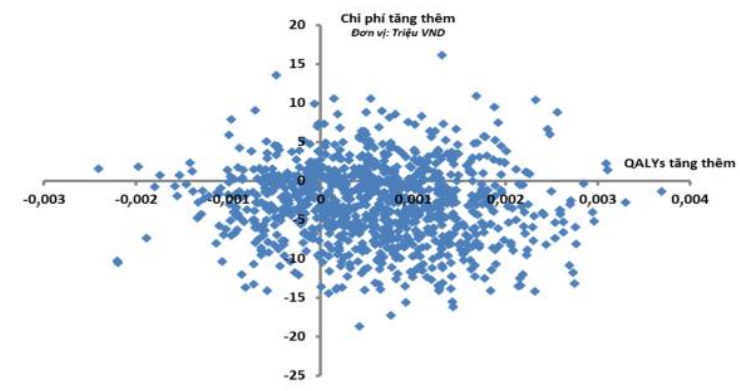

Hình 3. Mặt phẳng chi phí-hiệu quả của Budesonide/Formoterol khi cần vS. ICS liều thâp bid+SABA

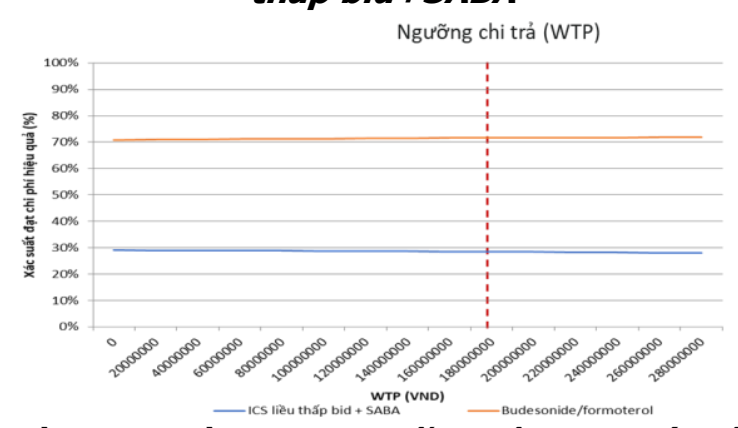

Hình 4. Đường cong chấp nhận chi phí-hiệu quả của Budesonide/Formoterol khi cân so với ICS+SABA

Với ngưỡng chi trả được đề xuất bởi WHO là 3 lần giá trị thu nhập bình quân đầu người cho một QALY tăng thêm, tương đương 187,7 triệu/QALY tăng thêm tại Việt Nam (Nguồn: GDP bình quân đâu người năm 2019, Tổng cục Thống 
kê), Budesonide/Formoterol khi cần có xác suất đạt chi phí-hiệu quả trên 70\% (Hình 4).

\section{BÀN LUẦN}

Nghiên cứu thực hiện phân tích chi phí-hiệu quả của Budesonide/Formoterol khi cần so với ICS+SABA đối với bệnh nhân HPQ nhẹ tại Việt Nam. Kết quả cho thấy Budesonide/Formoterol khi cần vượt trội so với phác đồ so sánh, giúp tránh được 0,44 đợt cấp $\mathrm{HPQ}$ năng, giảm chi phí (2.632.464VND) và tăng hiệu quả $(0,0006$ QALYs). Kết quả phân tích độ nhạy một chiều và độ nhạy xác suất đều khẳng định trong đa số trường hợp phân tích, Budesonide/Formoterol khi cần đều đạt chi phí-hiệu quả. Với ngưỡng chi trả được đề xuất bởi WHO là 3 lần giá trị thu nhập bình quân đầu người cho một QALY tăng thêm, Budesonide/Formoterol khi cần có xác suất đạt chi phí-hiệu quả là trên $70 \%$.

Kết quả này tương tự với các nghiên cứu phân tích kinh tế dược khác trên thế giới. Cụ thể, trong một nghiên cứu năm 2020 tại Anh, Budesonide/Formoterol khi cần giúp tiết kiệm chi phí là 293 bảng Anh trên một bệnh nhân và tăng thêm 0,001 QALYs so với phác đồ ICS+SABA [7]. Từ quan điểm cơ quan chi trả tại Anh với ngưỡng chi trả 20.000 bảng Ảnh/QALY, Budesonide/Formoterol khi cần được cho là đạt chi phí-hiệu quả. Tương tự, nghiên cứu tại Hà Lan (2009) cho thấy nhóm người bệnh sử dụng Budesonide/Formoterol giúp tiết kiệm 134,8 Euro [KTC 95\%: 44,9 đến 439,5] so với nhóm sử dụng ICS+SABA [8]. Trong đó, chi phí thuốc tiết kiệm được 36,1 Euro [KTC 95\%: 70,8 đến 161,1] và chi phí cho đợt cấp $\mathrm{HPQ}$ tiết kiệm được 73,9 Euro [KTC 95\%: 5,2 đến 219,2] cho mổi bênh nhân ở nhóm Budesonide/Formoterol so với phác đồ so sánh.

Một trong những vấn đề quan trọng trong thực tế lâm sàng điều trị HPQ tại Việt Nam cũng như các nước khác trên thế giới là mức tuân thủ điều trị của người bệnh. Các ước lượng của mô hình nghiên cứu dựa trên giả định mức độ tuân thủ của các phác đồ là như nhau. Do đó trong thực tế điều trị, ước lượng giá trị ICER có thể thay đổi theo hướng tốt hơn cho Budesonide/Formoterol.

Nghiên cứu này được thực hiện dựa trên dữ liệu lâm sàng từ nghiên cứu SYGMA $2[3,4]$, một nghiên cứu RCT đa trung tâm đa quốc gia trong đó có bao gồm nhóm dân số là người Việt Nam. Việc xây dựng dân số đầu vào của mô hình dựa trền đặc điểm dịch tễ bệnh dân số Việt Nam trong SYGMA 2 đã góp phần tăng tính sát thực của kết quả mô hình với thực tế điều trị HPQ tại Việt Nam.

\section{KẾT LUÂ̂N}

Từ quan điểm của cơ quan chi trả, Budesonide/Formoterol khi cần giúp giảm chi phí và tăng chất lượng sống của người bệnh so với phác đồ ICS liêuu thấp (ngày hai lần) phối hợp với SABA khi cần tại Việt Nam. Sử dụng Budesonide/Formoterol khi cần mang lại lợi ích về giảm thiểu chi phí và nâng cao chất lượng sống đối người bệnh HPQ nhẹ.

\section{LờI CẢM ƠN}

Nghiên cứu được thực hiện với sự tài trợ của Công ty TNHH AstraZeneca Việt Nam. Mọi quan điểm, kết quả nghiên cứu được trình bày ở đây là của nhóm nghiên cứu, độc lập với Công ty TNHH AstraZeneca Việt Nam.

\section{TÀI LIỆ THAM KHẢO}

1. Soriano JB, Kendrick PJ, Paulson KR, Gupta V, Abrams EM, Adedoyin RA, et al. Prevalence and attributable health burden of chronic respiratory diseases, 1990-2017: a systematic analysis for the Global Burden of Disease Study 2017. Lancet Respir Med. 2020;8(6):585-96.

2. Trân Thúy Hanh, Nguyễn Văn Đoàn, và cs Dịch tễ học và tình hình kiểm soát hen phế quản ở người trưởng thành Việt Nam. Trung tâm đào tạo và chỉ đạo tuyến Bạch Mai. 2011.

3. Nguyễn Viết Nhung, Nguyễn Đình Duy, Võ Đức Chiến, Lê Thị Thu Hương, Vũ Văn Thành. Hiệu quả, an toàn của Budesonide/Formoterol $(160 / 4,5 \mu \mathrm{g})$ Turbuhaler khi cần trên bệnh nhân hen nhẹ ( $\geq 12$ tuổi) ở Việt Nam: kết quả từ nghiên cứu SYGGMA 1\&2. Tạp chí $Y$ học lâm sàng. 2020;118(1):101-8.

4. Bateman ED, Reddel HK, O'Byrne PM, Barnes PJ, Zhong N, Keen $\mathbf{C}_{\text {, }}$ et al. As-Needed Budesonide-Formoterol versus Maintenance Budesonide in Mild Asthma. N Engl J Med. 2018;378 (20):1877-87.

5. Cục Quản lý Dược - Bộ Y tế. Dịch vụ công - Tra cứu giá thuốc. 2021.

6. Phạm Huy Tuấn Kiệt, Vũ Văn Giáp, Nguyễn Thị Thanh Hà. Chi phí y tế trực tiếp trong điêu trị hen theo phân loại GINA dựa trên dữ liệu lớn từ Bảo hiểm y tế Việt Nam năm 2019. Tạp chí Y học Việt Nam. 2021;503 (2):169-72.

7. FitzGerald JM, Arnetorp S, Smare C, Gibson D, Coulton K, Hounsell $\mathbf{K}$, et al. The costeffectiveness of as-needed budesonide/formoterol versus low-dose inhaled corticosteroid maintenance therapy in patients with mild asthma in the UK. Respir Med. 2020;171:106079.

8. Goossens LMA, Riemersma RA, Postma DS, Van Der Molen T, Rutten-Van Mölken MPMH. An economic evaluation of budesonide/formoterol for maintenance and reliever treatment in asthma in general practice. Adv Ther. 2009;26(9):872-85. 